This is the final submitted version of Masoli JAH, Todd OM, Clark CE. Systolic blood pressure and outcomes in frail older adults. Br J Hosp Med. 2021. https://doi.org/10.12968/ hmed.2021.0181 published online $26^{\text {th }}$ May 2021

\title{
Systolic blood pressure and outcomes in frail older adults
}

Jane AH Masoli 1,2, Oliver M Todd 3,4, Christopher E Clark 1

1 College of Medicine and Health, University of Exeter Medical School, UK

2 Department of Healthcare for Older People, Royal Devon and Exeter NHS Foundation Trust, Exeter, UK

3 Academic Unit for Ageing and Stroke Research, University of Leeds, Leeds, UK

4 Bradford Institute for Health Research, Bradford, UK

Correspondence to: Jane AH Masoli (j.masoli@exeter.ac.uk)

\section{Standfirst}

Hypertension is diagnosed in the majority of older people with frailty, in whom blood pressure prognosis is not well understood. We describe recent evidence on blood pressure and outcomes in older people with frailty.

\section{Background}

Hypertension is estimated to affect $75 \%$ of people older than 80 years of age (Benjamin et al, 2017). Approximately one-third of people over the age of 80 years have frailty (Gale et al, 2015). There are good reasons to consider that the balance of risks and benefits associated with treating hypertension may be different in the context of frailty.

Strong evidence exists for the treatment of hypertension in older adults, irrespective of chronological age, from two major international randomised clinical trials. Hypertension in the Very Elderly Trial (HYVET) (Bejan-Angoulvant et al, 2010) demonstrated benefit in treating systolic blood pressure to $<150 \mathrm{mmHg}$ to reduce stroke. The SPRINT trial (Williamson et al, 2016) showed that all-cause mortality and composite cardiovascular outcomes all reduced in those treated to a systolic blood pressure target $<120 \mathrm{mmHg}$ compared to those treated to a target of $<140 \mathrm{mmHg}$. However, both trials excluded people with multimorbidity, polypharmacy and frailty (Sheppard et al, 2020), and therefore it remains unclear whether trial findings have application to a significant proportion of older people.

Synthesis of traditional observational cohort studies has demonstrated that the association between systolic blood pressure and mortality varies with frailty status. Compared to older adults with a systolic blood pressure $>140 \mathrm{mmHg}$, those with a systolic blood pressure $\leq 140 \mathrm{mmHg}$ had reduced all-cause mortality if they were not frail. However, there was no association between systolic blood pressure and all-cause mortality in older people with frailty (Todd et al, 2019). These findings are difficult to apply to clinical practice. Many older people will have been excluded as a result of the traditional cohort study design. Systolic blood pressure and frailty were dichotomised, whereas in reality the risk associated with each exists on a continuum.

\section{Study summary}

The authors report a recently published observational study that tested the prognosis according to systolic blood pressure in 415980 older adults (mean age of 79.5 years) (Masoli et al, 2020), inclusive of those with multimorbidity and frailty. Data were from the Clinical Practice Research Datalink, with electronic primary healthcare records linked to hospital and mortality outcomes. The analyses were stratified by frailty 
status, established using the electronic frailty index cumulative deficit model (Clegg et al, 2016), which assigns categories of non-frail, mild, moderate and severe frailty based on specified 'deficits' within the primary care record.

The study tested associations between the baseline systolic blood pressure (median of all blood pressure readings over a 3-year period) and cardiovascular and mortality outcomes. Systolic blood pressure $<130 \mathrm{mmHg}$ and systolic blood pressure $>150 \mathrm{mmHg}$ were associated with increased risk of cardiovascular outcomes (stroke, myocardial infarction or cardiovascular procedure, heart failure) compared with a reference range of 130-139 mmHg. With frailty and in all aged over 85 years, hypertension (defined by a systolic blood pressure $>140 \mathrm{mmHg}$ ) was not associated with increased mortality compared to systolic blood pressure 130-139 mmHg (Masoli et al, 2020). These data suggest a possible protective effect of higher systolic blood pressure in people with frailty and in people aged over 85 years. However, the possibility that findings could be explained by reverse causality cannot be excluded, ie low systolic blood pressure in the context of disease burden may represent a marker of proximity to death, as blood pressure declines for over 15 years before death in older people (Delgado et al, 2018).

Figure 1. The association between systolic blood pressure and all-cause mortality stratified by age group and frailty, adapted from Masoli et al (2020). The original article includes detailed confidence intervals, adjustments and cardiovascular outcomes.

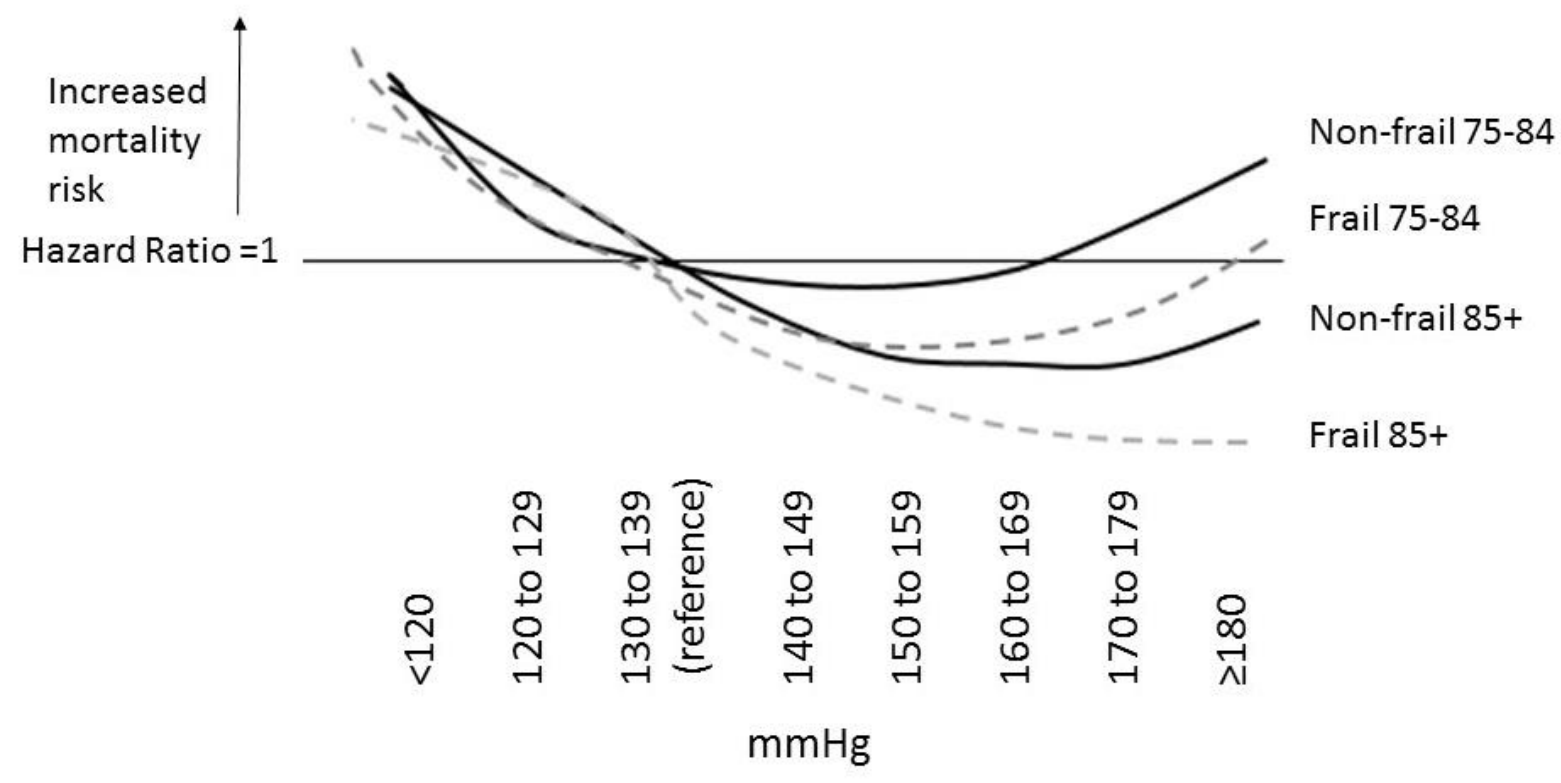

\section{Study strengths and limitations}

Observational studies of routine electronic healthcare data enable the inclusion of older adults who have advanced frailty, cognitive decline or physical limitations, all of which normally represent barriers to participation in research. The large cohort size enabled estimations of outcomes by narrow blood pressure categories of $10 \mathrm{mmHg}$, and analyses were stratified by frailty status within age groups of 75-84 years and over 85 years. The inclusion of non-fatal cardiovascular outcomes is a further key strength.

Sensitivity analyses found that associations between blood pressure and outcomes persisted despite the exclusion from the analysis of patients with cancer, patients within the last 6 months of life and patients with heart failure. Prescribing practices for hypertension in older adults will vary and may change with frailty. It is a limitation that antihypertensive prescribing is not described or adjusted for in the study. However, a 
previous study using Clinical Practice Research Datalink data showed similar outcomes despite adjustment for antihypertensive treatment status (Ravindrarajah et al, 2017).

Routinely recorded blood pressure is aligned with criteria used in clinical decision making in older people in primary care, and therefore results are generalisable to that setting. However, blood pressure measures from routine clinical data may not reflect a patient's true blood pressure: masked hypotension, postural hypotension and white coat hypertension can all be missed by an 'office' recording. Furthermore, recordings may have been undertaken during periods of acute illness and this may have influenced associations with outcomes.

Frailty in this study was measured using the electronic frailty index, which is accessible to the majority of GPs in the UK, and this improves the external validity of the study findings. Yet the electronic frailty index does not correlate exactly with other measures of frailty, and the degree of discordance could have significant impact on an individual's classification as frail or not frail.

\section{Clinical context and implications}

Internationally, hypertension guidelines differ on the optimal systolic blood pressure target for older adults, varying between $<130 \mathrm{mmHg}$ and $<150 \mathrm{mmHg}$. In the UK, the National Institute for Health and Care Excellence (NICE) hypertension guidelines (updated in 2019) found insufficient evidence to reduce the target in adults over 80 years from $<150 \mathrm{mmHg}$ (NICE, 2019). Several hypertension guidelines, including NICE, recommend clinical judgement be exercised in the context of frailty to weigh up benefits vs risks of hypertension treatment. However, there is little evidence to support specific approaches.

An important finding of this study is the association between systolic blood pressure $<130 \mathrm{mmHg}$ and increased mortality in all people over 75 years old, irrespective of frailty status. This finding supports clinicians taking a more cautious approach to avoid very low blood pressure in older people. The study also shows that in people with frailty and in the very old (over 85 years), risk profiles associated with hypertension may be different from the younger and less frail populations which are better represented in trials.

\section{Conclusions}

The optimal management of hypertension in patients who also have frailty or multimorbidity remains uncertain. In a study population representative of all older people seen in primary care, low systolic blood pressure was associated with increased mortality and, while hypertension increased cardiovascular risk, this was not associated with excess mortality in patients with frailty or patients aged over 85 years. More research is needed to better understand the prognostic role of frailty in hypertension management and to support clinical decision making.

\section{Funding}

Dr Jane Masoli is funded by the National Institute for Health Research (NIHR) (NIHR Development and Skills Enhancement Fellowship, NIHR301445). This publication presents independent research funded by the NIHR. The views expressed are those of the author(s) and not necessarily those of the NHS, the NIHR or the Department of Health and Social Care.

\section{Key points}

- NICE guidelines recommend treating blood pressure in adults aged over 80 years to a target of $<150 / 90 \mathrm{mmHg}$ and to 'use clinical judgement' in the context of frailty or multimorbidity.

- There is evidence from biological and epidemiological research to indicate that the relationship between systolic blood pressure and associated cardiovascular risk may be different in the context of frailty. 
- For older people without frailty and multimorbidity, systolic blood pressure should be treated irrespective of chronological age, while avoiding overtreatment resulting in hypotension.

- For older people with frailty and multimorbidity, there is an absence of evidence to directly inform management and a holistic and person-centred approach is recommended.

\section{References}

Bejan-Angoulvant T, Saadatian-Elahi M, Wright JM et al. Treatment of hypertension in patients 80 years and older: the lower the better? A meta-analysis of randomized controlled trials. J Hypertension.

2010;28(7):1366-1372. https://doi.org/10.1097/HJH.0b013e328339f9c5

Benjamin EJ, Blaha MJ, Chiuve SE et al; American Heart Association Statistics Committee and Stroke Statistics Subcommittee. Heart Disease and Stroke Statistics-2017 Update: A Report From the American Heart Association. Circulation. 2017;135(10):e146-e603. https://doi.org/10.1161/CIR.0000000000000485

Clegg A, Bates C, Young J et al. Development and validation of an electronic frailty index using routine primary care electronic health record data. Age Ageing. 2016;45(3):353-360. https://doi.org/10.1093/ageing/afw039

Delgado J, Bowman K, Ble A et al. Blood pressure trajectories in the 20 years before death. JAMA Intern Med. 2018;178(1):93. https://doi.org/10.1001/jamainternmed.2017.7023

Gale CR, Cooper C, Sayer AA. Prevalence of frailty and disability: findings from the English Longitudinal Study of Ageing. Age Ageing. 2015;44(1):162-165. https://doi.org/10.1093/ageing/afu148

Masoli JAH, Delgado J, Pilling L et al. Blood pressure in frail older adults: associations with cardiovascular outcomes and all-cause mortality. Age Ageing. 2020;49(5):807-813. https://doi.org/10.1093/ageing/afaa028

National Institute for Health and Care Excellence. Hypertension in adults: diagnosis and management. NG136. 2019. https://www.nice.org.uk/guidance/ng136

Ravindrarajah R, Hazra NC, Hamada S et al. Systolic blood pressure trajectory, frailty, and all-cause mortality and 80 years of age: cohort study using electronic health records. Circulation. 2017;135(24):2357-2368. https://doi.org/10.1161/CIRCULATIONAHA.116.026687

Sheppard JP, Lown M, Burt J et al. Generalizability of blood pressure lowering trials to older patients: crosssectional analysis. J Am Geriatr Soc. 2020;68(11):2508-2515. https://doi.org/10.1111/jgs.16749

Todd OM, Wilkinson C, Hale $\mathrm{M}$ et al. Is the association between blood pressure and mortality in older adults different with frailty? A systematic review and meta-analysis. Age Ageing. 2019;48(5):627-635.

https://doi.org/10.1093/ageing/afz072

Williamson JD, Supiano MA, Applegate WB, for the SPRINT Research Group et al. Intensive vs standard blood pressure control and cardiovascular disease outcomes in adults aged $\geq 75$ years. JAMA. 2016;315(24):2673. https://doi.org/10.1001/jama.2016.7050 